\title{
A Multilevel Space-Time Varying Coefficient Model: The Equity of Service Accessibility
}

- Supplemental Material -

\section{Supplemental Material 1: Varying-Coefficient model}

Decomposition of the Design Matrix. In this paper, we decompose the space-time varying coefficients using a semi-parametric model into global separable space and time effects along with space-time deviations from the global effects which are intrinsically local and account for the interaction between space and time:

$$
\gamma_{r}(t, s)=\alpha_{r}(t)+\beta_{r}(s)+\sum_{m=1}^{M_{r}} \sum_{n=1}^{N_{r}} \nu_{r, m n} K_{t e m p}\left(\left|t-\kappa_{m}^{(T)}\right|\right) K_{s p}\left(\left\|s-\kappa_{n}^{(S)}\right\|\right) .
$$

where $\gamma_{r}(t, s)$ contains three random terms as follows

$\sum_{m=1}^{M} u_{r, m} K_{t e m p}\left(\left|t-\kappa_{m}^{(T)}\right|\right), \sum_{n=1}^{N} v_{r, n} K_{s p}\left(\left\|s-\kappa_{n}^{(S)}\right\|\right), \sum_{m=1}^{M} \sum_{n=1}^{N} \nu_{r, m n} K_{t e m p}\left(\left|t-\kappa_{m}^{(T)}\right|\right) K_{s p}\left(\left\|s-\kappa_{n}^{(S)}\right\|\right)$.

Following the notation in Section 4.1, we can represent $\gamma_{r}(t, s)$ as $\mathbf{X}_{\mathbf{r}} \boldsymbol{\beta}_{r}+\mathbf{Z}_{\mathbf{r}} \mathbf{u}_{\mathbf{r}}$ where $\beta_{r}$ are the fixed effects and $u_{r}$ are the random effects corresponding to $\gamma_{r}(t, s)$. Denote

$$
\begin{aligned}
\mathbf{Z}_{\mathbf{0}}= & {\left[K_{\text {temp }}\left(\left|t_{i}-\kappa_{m}^{(T)}\right|\right) K_{s p}\left(\left\|s_{j}-\kappa_{n}^{(S)}\right\|\right)\right.} \\
& \left.K_{\text {temp }}\left(\left|t_{i}-\kappa_{m}^{(T)}\right|\right) K_{s p}\left(\left\|s_{j}-\kappa_{n}^{(S)}\right\|\right)\right]_{i=1, \ldots, T, j=1, \ldots, S ; n=1, \ldots, N, m=1, \ldots, M}
\end{aligned}
$$

where $Z_{r}$ is the vector multiplication of $Z_{0}$ and $\left\{X_{r}\left(t_{i}, s_{j}\right)\right\}_{i=1, \ldots, T, j=1, \ldots, S}$. We can equivalently write $Z_{0}$ as

$$
\begin{aligned}
\mathbf{Z}_{\mathbf{0}}= & {\left[\mathbf{1}_{S} \otimes K_{\text {temp }}\left(\left|t_{i}-\kappa_{m}^{(T)}\right|\right) K_{s p}\left(\left\|s_{j}-\kappa_{n}^{(S)}\right\|\right) \otimes \mathbf{1}_{T}\right.} \\
& \left.K_{s p}\left(\left\|s_{j}-\kappa_{n}^{(S)}\right\|\right) \otimes K_{t e m p}\left(\left|t_{i}-\kappa_{m}^{(T)}\right|\right)\right]_{i=1, \ldots, T, j=1, \ldots, S ; n=1, \ldots, N, m=1, \ldots, M}
\end{aligned}
$$

where $T$ is the number of temporal design points and $S$ is the number of spatial design points. In this decomposition, $\mathbf{Z}_{\mathbf{0}}$ has $T S$ rows and $M T+N S+M \times N$ columns. For computational efficiency, we replace $Z_{0}$ by

$$
\left.\mathbf{Z}_{\mathbf{0}}^{\prime}=\left[\begin{array}{ll}
\mathbf{1}_{S} & K_{s p}\left(\left\|s_{j}-\kappa_{n}^{(S)}\right\|\right)
\end{array}\right] \otimes\left[\mathbf{1}_{T} K_{t e m p}\left(\left|t_{i}-\kappa_{m}^{(T)}\right|\right)\right]\right]_{i=1, \ldots, T, j=1, \ldots, S ; n=1, \ldots, N, m=1, \ldots, M}
$$

where $\mathbf{Z}_{\mathbf{0}}^{\prime}$ has $T \times S$ rows and $M T+N S+M \times N+T S$ columns. The difference between $\mathbf{Z}_{\mathbf{0}}^{\prime}$ and $\mathbf{Z}_{\mathbf{0}}$ is that $\mathbf{Z}_{\mathbf{0}}^{\prime}$ has extra $T \times S$ columns. In the estimation procedure, we assume that the coefficients corresponding to the extra columns are fixed and equal to zero. Consequently, we ensure that the estimation using the decomposition $\mathbf{Z}_{\mathbf{0}}^{\prime} \mathbf{u}_{\mathbf{r}}$ is equivalent to the estimation using the decomposition $\mathbf{Z}_{\mathbf{0}} \mathbf{u}_{\mathbf{r}}$. 


\section{Supplemental Material 2: Varying-Coefficient model}

Derivation of the Confidence Bands. In this appendix, we derive confidence bands for time-varying coefficients for a model with a single predictor. For a model with multiple predictors, we simply apply the formula derived here for each predictor separately. We do not provide the confidence bands for spatial-varying coefficients since they simply follow a similar derivation as for the temporal-varying coefficients.

We start with a simple model $Y_{i}=f\left(x_{i}\right)+\epsilon_{i}$ with estimated regression function

$$
\hat{f}\left(x_{i}\right)=\hat{\tau}_{0}\left(t_{i}\right)+\hat{\tau}_{1}\left(t_{i}\right) x_{i}=\mathbf{X} \hat{\boldsymbol{\beta}}+\mathbf{Z} \hat{\mathbf{u}}
$$

where $\mathbf{X}=\left[\begin{array}{ll}\mathbf{X}^{(\mathbf{0})} & \mathbf{X}^{(\mathbf{1})}\end{array}\right]$ and $\mathbf{Z}=\left[\begin{array}{ll}\mathbf{Z}^{(\mathbf{0})} & \mathbf{Z}^{(\mathbf{1})}\end{array}\right]$. Denote $g=\tau_{1}\left(t_{i}\right) x_{i}$. Our problem reduces to construction of confidence bands for the varying-coefficients $\hat{\tau}_{0}(t)$ and $\tau_{1}(t)$. Denote

$$
\begin{gathered}
\operatorname{Cov}(\boldsymbol{\epsilon})=\sigma_{\epsilon}^{2} \mathbf{I}, \quad \operatorname{Cov}(\mathbf{u})=\left[\begin{array}{cc}
\sigma_{(0)}^{2} \mathbf{I} & \mathbf{0} \\
\mathbf{0} & \sigma_{(1)}^{2} \mathbf{I}
\end{array}\right], \text { and } \\
\mathbf{M}=E\left\{\left[\begin{array}{c}
\hat{\beta}^{(0)}-\beta^{(0)} \\
\hat{u}^{(0)}-u^{(0)} \\
\hat{\beta}^{(1)}-\beta^{(1)} \\
\hat{u}^{(1)}-u^{(1)}
\end{array}\right]\left[\begin{array}{c}
\hat{\beta}^{(0)}-\beta^{(0)} \\
\hat{u}^{(0)}-u^{(0)} \\
\hat{\beta}^{(1)}-\beta^{(1)} \\
\hat{u}^{(1)}-u^{(1)}
\end{array}\right]\right\}
\end{gathered}
$$

Using the notation above, we further have

$$
\mathbf{M}=\sigma_{\epsilon}^{2}\left[\begin{array}{cccc}
\mathbf{X}^{\prime(\mathbf{0})} \mathbf{X}^{(\mathbf{0})} & \mathbf{X}^{\prime(\mathbf{0})} \mathbf{Z}^{(\mathbf{0})} & \mathbf{X}^{\prime(\mathbf{0})} \mathbf{X}^{(\mathbf{1})} & \mathbf{X}^{(\mathbf{0})} \mathbf{Z}^{(\mathbf{1})} \\
\mathbf{Z}^{\prime(\mathbf{0})} \mathbf{X}^{(\mathbf{0})} & \mathbf{Z}^{(\mathbf{0})} \mathbf{Z}^{(\mathbf{0})}+\sigma_{\epsilon}^{2} / \sigma_{(0)}^{2} \mathbf{I} & \mathbf{Z}^{\prime(\mathbf{0})} \mathbf{X}^{(\mathbf{1})} & \mathbf{Z}^{\prime(\mathbf{0})} \mathbf{Z}^{(\mathbf{1})} \\
\mathbf{X}^{\prime(\mathbf{1})} \mathbf{X}^{(\mathbf{0})} & \mathbf{X}^{\prime(\mathbf{1})} \mathbf{Z}^{(\mathbf{0})} & \mathbf{X}^{\prime(\mathbf{1})} \mathbf{X}^{(\mathbf{1})} & \mathbf{X}^{\prime(\mathbf{1})} \mathbf{Z}^{(\mathbf{1})} \\
\mathbf{Z}^{\prime(\mathbf{1})} \mathbf{X}^{(\mathbf{0})} & \mathbf{Z}^{\prime(\mathbf{1})} \mathbf{Z}^{(\mathbf{0})} & \mathbf{Z}^{\prime(\mathbf{1})} \mathbf{X}^{(\mathbf{1})} & \mathbf{Z}^{\prime(\mathbf{1})} \mathbf{Z}^{(\mathbf{1})}+\sigma_{\epsilon}^{2} / \sigma_{(1)}^{2} \mathbf{I}
\end{array}\right]^{-1}
$$

and

$$
\widehat{\text { st.dev }} \cdot\{\hat{f}(x)-f(x)\}=\sqrt{\left[\begin{array}{llllll}
\mathbf{X}^{(\mathbf{0})} & \mathbf{Z}^{(\mathbf{0})} & \mathbf{X}^{(\mathbf{1})} & \mathbf{Z}^{(\mathbf{1})}
\end{array}\right] \mathbf{M}^{\prime}\left[\begin{array}{llll}
\mathbf{X}^{(\mathbf{0})} & \mathbf{Z}^{(\mathbf{0})} & \mathbf{X}^{(\mathbf{1})} & \mathbf{Z}^{(\mathbf{1})}
\end{array}\right]^{\prime}} .
$$

In order to derive confidence bands for both $\tau_{0}(t)$ and $\tau_{1}(t)$, we write the matrix $\mathbf{M}$ as follows.

$$
\begin{aligned}
& \mathbf{M}=\left[\begin{array}{ll}
\mathbf{A} & \mathbf{B} \\
\mathbf{C} & \mathbf{D}
\end{array}\right]^{-1} \text { where } \\
& \mathbf{A}=\left[\begin{array}{cc}
\mathbf{X}^{\prime(\mathbf{0})} \mathbf{X}^{(\mathbf{0})} & \mathbf{X}^{\prime(\mathbf{0})} \mathbf{Z}^{(\mathbf{0})} \\
\mathbf{Z}^{\prime(\mathbf{0})} \mathbf{X}^{(\mathbf{0})} & \mathbf{Z}^{\prime(\mathbf{0})} \mathbf{Z}^{(\mathbf{0})}+\sigma_{\epsilon}^{2} / \sigma_{(0)}^{2} \mathbf{I}
\end{array}\right] \quad \mathbf{B}=\left[\begin{array}{cc}
\mathbf{X}^{(\mathbf{0})} \mathbf{X}^{(\mathbf{1})} & \mathbf{X}^{(\mathbf{0})} \mathbf{Z}^{(\mathbf{1})} \\
\mathbf{Z}^{\prime(\mathbf{0})} \mathbf{X}^{(\mathbf{1})} & \mathbf{Z}^{(\mathbf{0})} \mathbf{Z}^{(\mathbf{1})}
\end{array}\right] \\
& \mathbf{C}=\left[\begin{array}{ll}
\mathbf{X}^{\prime(\mathbf{1})} \mathbf{X}^{(\mathbf{0})} & \mathbf{X}^{\prime(\mathbf{1})} \mathbf{Z}^{(\mathbf{0})} \\
\mathbf{Z}^{\prime(\mathbf{1})} \mathbf{X}^{(\mathbf{0})} & \mathbf{Z}^{\prime(\mathbf{1})} \mathbf{Z}^{(\mathbf{0})}
\end{array}\right] \quad \mathbf{D}=\left[\begin{array}{cc}
\mathbf{X}^{\prime(\mathbf{1})} \mathbf{X}^{(\mathbf{1})} & \mathbf{X}^{\prime(\mathbf{1})} \mathbf{Z}^{(\mathbf{1})} \\
\mathbf{Z}^{\prime(\mathbf{1})} \mathbf{X}^{(\mathbf{1})} & \mathbf{Z}^{\prime(\mathbf{1})} \mathbf{Z}^{(\mathbf{1})}+\sigma_{\epsilon}^{2} / \sigma_{(1)}^{2} \mathbf{I}
\end{array}\right]
\end{aligned}
$$


Since matrices can be inverted blockwise by using the following analytic inversion formula:

$$
\left[\begin{array}{ll}
\mathbf{A} & \mathbf{B} \\
\mathbf{C} & \mathbf{D}
\end{array}\right]^{-1}=\left[\begin{array}{cc}
\mathbf{A}^{-1}+\mathbf{A}^{-1} \mathbf{B}\left(\mathbf{D}-\mathbf{C A}^{-1} \mathbf{B}\right)^{-1} \mathbf{C A}^{-1} & -\mathbf{A}^{-1} \mathbf{B}\left(\mathbf{D}-\mathbf{C A}^{-1} \mathbf{B}\right)^{-1} \\
-\left(\mathbf{D}-\mathbf{C A}^{-1} \mathbf{B}\right)^{-1} \mathbf{C A}^{-1} & \left(\mathbf{D}-\mathbf{C A}^{-1} \mathbf{B}\right)^{-1}
\end{array}\right]
$$

we can directly derive the standard deviations of the estimators of $\tau_{0}(t)$ and $g(t)=\tau_{1}(t) X$ as below

$$
\begin{aligned}
& \widehat{\text { st.dev }} \cdot\left\{\hat{\tau}_{0}-\tau_{0}\right\} \\
= & \sigma_{\epsilon} \sqrt{\left[\mathbf{X}^{(\mathbf{0})} \mathbf{Z}^{(\mathbf{0})}\right]\left[\mathbf{A}^{-\mathbf{1}}+\mathbf{A}^{-\mathbf{1}} \mathbf{B}\left(\mathbf{D}-\mathbf{C A}^{-\mathbf{1}} \mathbf{B}\right)^{-\mathbf{1}} \mathbf{C A}^{-\mathbf{1}}\right]\left[\begin{array}{|}
\mathbf{X}^{(\mathbf{0})} & \mathbf{Z}^{(\mathbf{0})}
\end{array}\right]^{\prime}}
\end{aligned}
$$

and

$$
\begin{aligned}
& \widehat{\text { st.dev }} \cdot\{\hat{g}-g\} \\
= & \left.\sigma_{\epsilon} \sqrt{\left[\mathbf{X}^{(\mathbf{1})}\right.} \mathbf{Z}^{(\mathbf{1})}\right]\left[\left(\mathbf{D}-\mathbf{C A}^{-\mathbf{1}} \mathbf{B}\right)^{-\mathbf{1}}\right]\left[\begin{array}{ll}
\mathbf{X}^{(\mathbf{1})} & \mathbf{Z}^{(\mathbf{1})}
\end{array}\right]^{\prime} .
\end{aligned}
$$

Hence the confidence bands are

$$
\begin{aligned}
& \hat{\tau}_{0}(t) \pm \begin{cases}t d\left(1-\frac{\alpha}{2} ; d f_{\text {res }}\right) \widehat{\text { st.dev }} \cdot\left\{\hat{\tau}_{0}(t)-\tau_{0}(t)\right\} & \text { for small } n \\
z\left(1-\frac{\alpha}{2}\right) \widehat{\text { st.dev. }}\left\{\left\{\hat{\tau}_{0}(t)-\tau_{0}(t)\right\}\right. & \text { for large } n\end{cases} \\
& g \pm \begin{cases}t\left(1-\frac{\alpha}{2} ; d f_{\text {res }}\right) \widehat{\text { st.dev }} \cdot\{\hat{g}-g\} & \text { for small } n \\
z\left(1-\frac{\alpha}{2}\right) \widehat{\text { st.dev. }}\{\{\hat{g}-g\} & \text { for large } n\end{cases}
\end{aligned}
$$

where $t d(1-\alpha ; d f)$ and $z(1-\alpha)$ are the $1-\alpha$ quantile for the t-distribution, and respectively, for the normal distribution.

To obtain the confidence band for the varying coefficient $\tau_{1}$ we note that

$$
\hat{f}^{(1)}=\hat{\tau}_{1}\left(t_{i}\right) x_{i} \Longrightarrow \widehat{\operatorname{st} . \operatorname{dev}} \cdot\left\{\hat{f}^{(1)}-f^{(1)}\right\}=\widehat{\operatorname{st} . \operatorname{dev} \cdot}\left\{\hat{\tau}_{1}\left(t_{i}\right)-\tau_{1}\left(t_{i}\right)\right\} \sqrt{x_{i}^{2}} .
$$

Therefore, the confidence band for $\tau_{1}(t)$ at $t=t_{i}$ is

$$
\hat{\tau}_{1}\left(t_{i}\right) \pm \begin{cases}t\left(1-\frac{\alpha}{2} ; d f_{\text {res }}\right) \frac{\widehat{\text { st.dev. }\{\hat{g}-g\}}}{\sqrt{x_{i}^{2}}} & \text { for small } n \\ z\left(1-\frac{\alpha}{2}\right) \frac{\widehat{\text { st.dev. }\{\hat{g}-g\}}}{\sqrt{x_{i}^{2}}} & \text { for large } n\end{cases}
$$

\section{Supplemental Material 3: Varying-Coefficient model}

Simulation Study. In this section, we evaluate the estimation and inference procedures for the space-time varying coefficient model described in Section 2 of the accompanying manuscript. We simulate from the following model:

$Y\left(t_{i} ; s_{j}\right)=\left(\alpha_{1}\left(t_{i}\right)+\beta_{1}\left(s_{j}\right)+\gamma_{1}\left(t_{i}, s_{j}\right)\right) X_{1}\left(t_{i}, s_{j}\right)+\left(\alpha_{2}\left(t_{i}\right)+\beta_{2}\left(s_{j}\right)+\gamma_{2}\left(t_{i}, s_{j}\right)\right) X_{2}\left(t_{i}, s_{j}\right)+\epsilon_{i j}$ where 
- $t_{i}, i=1, \ldots, T(T=15)$ are equally spaced in the time domain $[0, T]$;

- $s_{j}, j=1, \ldots, S(S=300)$ are also equally spaced in the space domain $[-10,10]$;

- $X_{1}\left(t_{i}, s_{j}\right)$ and $X_{2}\left(t_{i}, s_{j}\right)$ for $i=1, \ldots, T$ and $j=1, \ldots, S$ are uniformly sampled $(U[2,3])$;

- The true temporal coefficients are:

$$
\begin{aligned}
& \alpha_{1}(t)=2-0.1 t \\
& \alpha_{2}(t)=2-\cos \left(\frac{(t-25) * \pi}{15}\right) ;
\end{aligned}
$$

- The true spatial coefficients are:

$$
\begin{aligned}
& \beta_{1}\left(s_{1}, s_{2}\right)=\frac{\left(\sqrt{s_{1}^{2}+s_{2}^{2}}\right)}{10} \text { or } \beta_{1}\left(s_{1}, s_{2}\right)=2 \\
& \beta_{2}\left(s_{1}, s_{2}\right)=\frac{3 \times \sin \left(\sqrt{s_{1}^{2}+s_{2}^{2}}\right)}{\sqrt{s_{1}^{2}+s_{2}^{2}}}
\end{aligned}
$$

- The true space-time interaction coefficients are decomposed as follows

$$
\gamma_{r}(t, s)=\sum_{m=1}^{4} \sum_{n=1}^{10} \nu_{r, i j} K_{t e m p}\left(t-\kappa_{r, m}^{(T)}\right) K_{s p}\left(s-\kappa_{r, n}^{(S)}\right)
$$

where for $r=1,2, \nu_{r, i j} \sim N(0,0.5)$ independently distributed, $\kappa_{r, m}^{(T)}, m=1, \ldots, 4$ are uniformly sampled from the time domain and $\kappa_{r, n}^{(S)}, n=1, \ldots, 10$ are uniformly sampled from the space domain;

- The error terms are assumed normally distributed with mean zero and standard deviation $\sigma=1$.

In Figure 1, we show the true and the estimated temporal coefficients along with their confidence bands and in Figure 2, we show the true and the estimated spatial coefficients from the application of the space-time varying coefficient model to one simulation data. The estimated coefficients accurately capture the shape of the true coefficients.

We also evaluate the coverage and the power of the shape evaluation procedure discussed in Section 2.3 of the accompanying paper. Specifically, we compute coverage for the shape evaluation of $\alpha_{1}(t)$ which is assumed linear and the power for the shape evaluation of $\alpha_{2}(t)$ which is assumed nonlinear. Moreover, we compute the coverage for the shape evaluation of $\beta_{1}(t)$ for the simulation setting when it is assumed constant and the power for shape evaluation of $\beta_{1}(t)$ and $\beta_{2}(t)$ for the simulation setting when they are assumed nonlinear.

We report coverage and power results for identifying the shape of the time-varying and space-varying components in Table 1 . We note that the accuracy measured by the power of the shape evaluation procedure is lower for the time-varying components than for the space-varying ones. Moreover, the power depends on how smooth and close to the null hypothesis the shape functions is: compare the power for $\beta_{1}(t)$ to the power for 


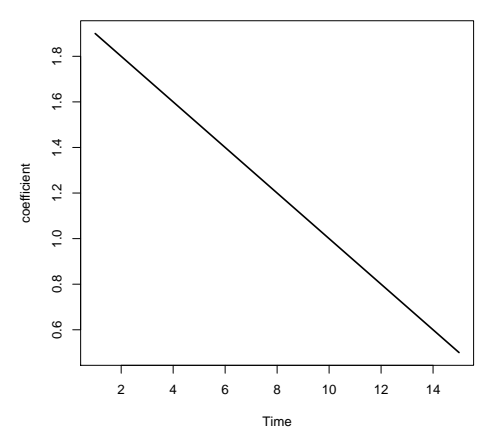

(a) True $\alpha_{1}(t)$

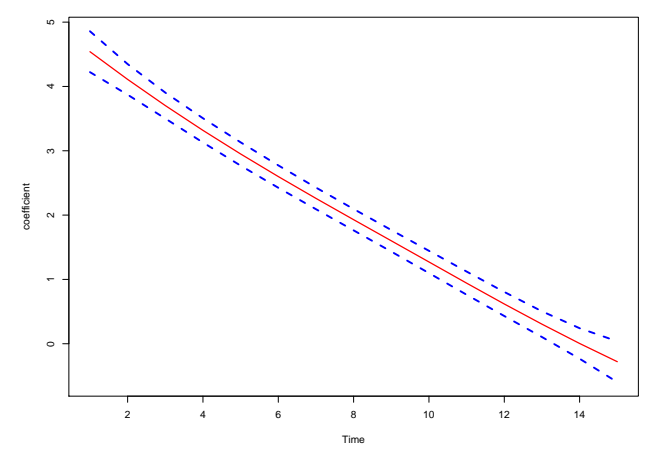

(c) Estimated $\alpha_{1}(t)$

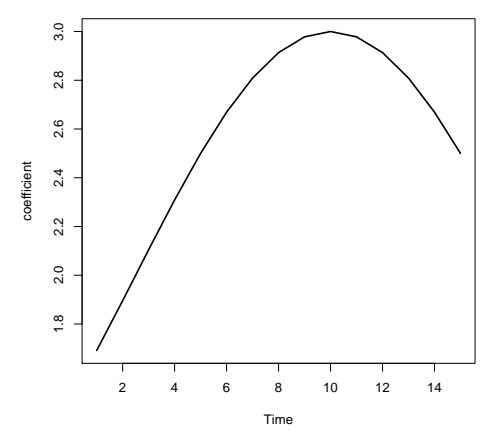

(b) True $\alpha_{2}(t)$

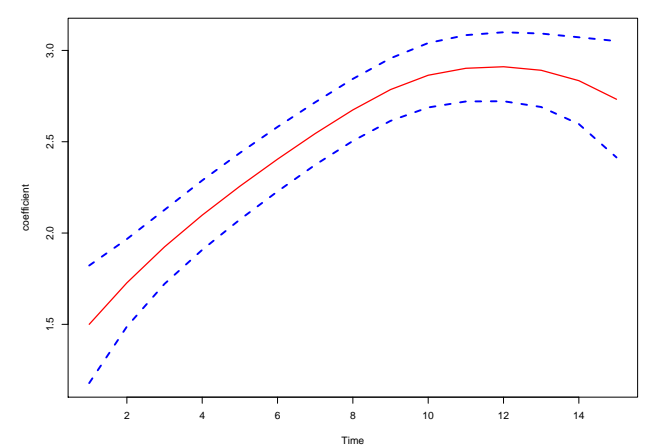

(d) Estimated $\alpha_{2}(t)$

Figure 1: True and Estimated time-varying coefficients.

$\beta_{2}(t)$. The coverage for testing the null hypothesis of linear $\alpha_{1}(t)$ is rather low; although the estimated function is approximately linear with some variations on the upper end, because the confidence bands are tight, the shape evaluation procedure will decide for rejecting the hypothesis of linear shape in about $20 \%$ of the simulations.

Table 1: Coverage and Power Evaluations.

\begin{tabular}{rrrrr}
\hline Coverage $\alpha_{1}(t)$ & Power $\alpha_{2}(t)$ & Coverage $\beta_{1}(t)$ & Power $\beta_{1}(t)$ & Power $\beta_{2}(t)$ \\
\hline 0.81 & 0.77 & 0.90 & 0.80 & 0.93 \\
\hline
\end{tabular}

\section{Supplemental Material 4: Varying-Coefficient model}

Proof of Proposition 2. In this appendix, we provide a brief reasoning for the Proposition 2 stated in Section 5.2. 


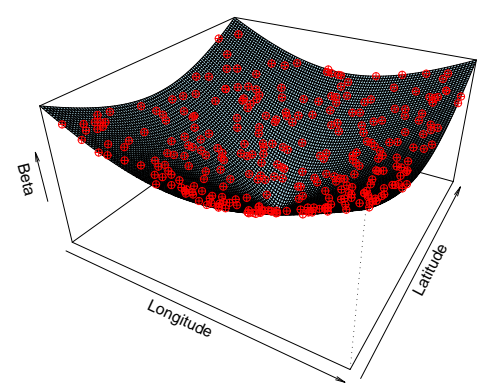

(a) True $\beta_{1}(t)$

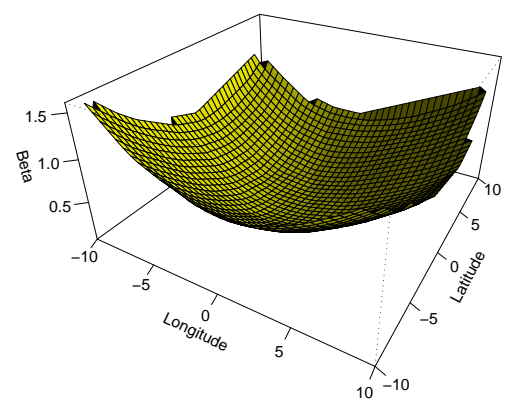

(c) Estimated $\beta_{1}(t)$

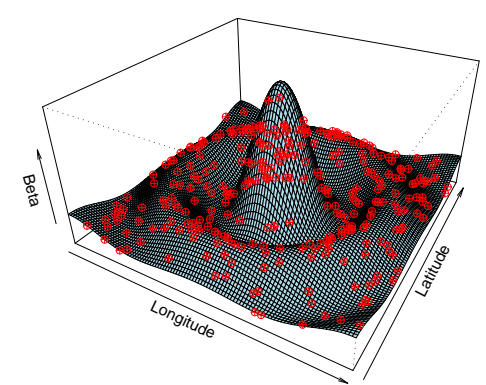

(b) True $\beta_{2}(t)$

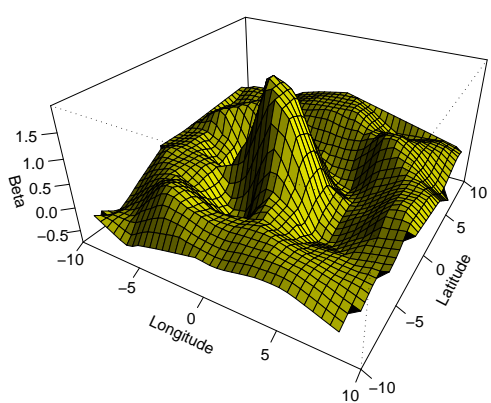

(d) Estimated $\beta_{2}(t)$

Figure 2: True and Estimated space-varying coefficients.

We first define the global fixed and effects corresponding to $\gamma_{r}(t, s)$ :

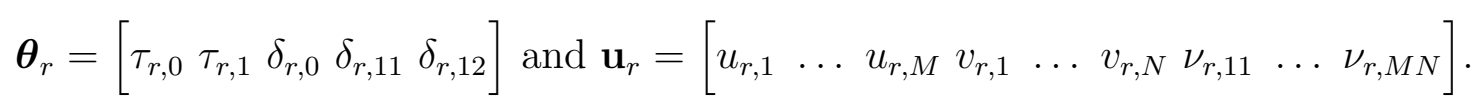

The corresponding design matrices for these coefficients $\mathbf{X}_{r}$ and $\mathbf{Z}_{r}$ are

$$
\begin{aligned}
& \mathbf{X}_{r}=\left\{X_{r}\left(t_{i}, s_{j}\right)\left[\begin{array}{lllll}
1 & t_{i} & 1 & s_{1 j} & s_{2 j}
\end{array}\right]\right\}_{i=1, \ldots, T, j=1, \ldots, S} \\
& \mathbf{Z}_{r}=\left\{X_{r}\left(t_{i}, s_{j}\right)\left[K_{t e m p}\left(\left|t_{i}-\kappa_{m, r}^{(T)}\right|\right) K_{s p}\left(\left\|s_{i}-\kappa_{n, r}^{(S)}\right\|\right)\right]\right\}_{i=1, \ldots, T, j=1, \ldots, S ; n=1, \ldots, N, m=1, \ldots, M}
\end{aligned}
$$

For the multilevel model, we have extra terms for the second level of hierarchy $\left(\eta_{r p}(t, s)\right)$ defined in the vector form for the $p$ th group by

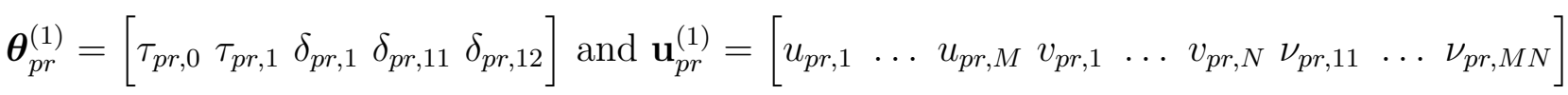


The corresponding design matrices for these coefficients are $\mathbf{X}_{p r}^{(1)}=\mathbf{X}_{r}$ and $\mathbf{Z}_{p r}^{(1)}$ with

$$
\mathbf{Z}_{p r}^{(1)}=\left\{X_{r}\left(t_{i}, s_{j}\right)\left[K_{t e m p}\left(\left|t_{i}-\kappa_{m, p}^{(T)}\right|\right) K_{s p}\left(\left\|s_{i}-\kappa_{n, p}^{(S)}\right\|\right) K_{t e m p}\left(\left|t_{i}-\kappa_{m, p}^{(T)}\right|\right) K_{s p}\left(\left\|s_{i}-\kappa_{n, p}^{(S)}\right\|\right)\right]\right\}_{i=1, \ldots, T, j=}
$$

We define the vectors corresponding to the fixed effects and the random effects for the $r$ th predictor

$$
\begin{aligned}
\Theta_{r} & =\left[\theta_{r} \ldots \theta_{r}\right] \text { and } \Theta_{r}^{(1)}=\left[\theta_{1 r}^{(1)} \ldots \theta_{P r}^{(1)}\right] \\
\mathbf{U}_{r} & =\left[\mathbf{u}_{r} \ldots \mathbf{u}_{r}\right] \text { and } \mathbf{U}_{r}^{(1)}=\left[\mathbf{u}_{1 r}^{(1)} \ldots \mathbf{u}_{P r}^{(1)}\right]
\end{aligned}
$$

The corresponding design matrices are:

$$
\begin{aligned}
\mathcal{X}_{r} & =\left[\mathbf{X}_{r} \ldots \mathbf{X}_{r}\right]^{\mathrm{T}} \text { and } \mathcal{X}_{r}^{(1)}=\text { block-diag }\left[\mathbf{X}_{p r}^{(1)}\right]_{p=1}^{P} \\
\mathcal{Z}_{r} & =\left[\mathbf{Z}_{r} \ldots \mathbf{Z}_{r}\right]^{\mathrm{T}} \text { and } \mathcal{Z}_{r}^{(1)}=\text { block-diag }\left[\mathbf{Z}_{p r}^{(1)}\right]_{p=1}^{P}
\end{aligned}
$$

The model in the matrix form becomes

$$
\mathbb{E}[Y \mid X]=\sum_{r=1}^{R}\left\{\mathcal{X}_{r} \Theta_{r}+\mathcal{Z}_{r} \mathbf{U}_{r}+\mathcal{X}_{r}^{(1)} \Theta_{r}^{(1)}+\mathcal{Z}_{r}^{(1)} \mathbf{U}_{r}^{(1)}\right\}
$$

The conditions on the temporal and spatial knots in Proposition 2 ensure that $\mathbf{Z}_{p r} \neq \mathbf{Z}_{p^{\prime} r}$ for $p=1, \ldots, P$. The equality would induce linear dependence between the columns of the full design matrix $\mathcal{Z}_{r}^{(1)}$.

\section{Supplemental Material 5: Case Study}

ESRI Data. For estimates before 2000, ESRI obtains the race and ethnicity estimates at the census tract level using the most current county-level estimates produced by the Census Bureau. County-level analysis is supplemented by estimates from the Census Bureau's Current Population Survey (CPS). Using the county level estimates combined with local data, forecasts are derived at the block level. From the block level database, forecasts can be retrieved for census tracts. For estimates after 2000, ESRI uses the most current data by race and Hispanic origin available by county and state from the Census Bureaus estimates and its American Community Survey (ACS).

The documentation complementing the ESRI data provides the following information about race and ethnicity variables:

Tracking intercensal population change by race is encumbered by the new reporting method in Census 2000. Race was reported as a multiple-choice item-not "one personone race" as reported in past censuses or estimates. The 2000 data are not directly comparable to 1990 Census data, or to any earlier estimates or projections. ESRI takes an innovative approach in analyzing this data to make effective use of the additional information from Census 2000. The Census Bureau released most race-related data by 
six single-race groups and one multiple-race group. ESRIs data preserve this format and enable a comparison of 1990 and 2000 data for six single races and one multiracial group. Assuming that the probability of reporting more than one race varies by race group and geographic area (as shown in Census 2000), ESRI estimates the number of likely multiple race reporters from 1990 Census data. The same approach is adopted for the population of Hispanic origin by race. Local trends in the distribution of the population by race and Hispanic origin are incorporated with current population estimates in the model. Forecasts by block group combine local changes in the distributions by race and projected change for counties. The last step controls block group distributions to county projections. Data are reported by Hispanic origin for seven race groups that are consistent with Census 2000 tabulations. From the block group database, forecasts can be retrieved for census tracts.

\section{Supplemental Material 6: Case Study}

Accessibility Maps: Atlanta. In this section, we present the accessibility maps for the highest populated area in Georgia: Atlanta and its surroundings. In Figure 3, we present the travel cost (without population density scaling) (a, b) and accessibility (with population density scaling) (c,d).

In Section 6.1 of the accompanying article we pointed out a series of differences between the travel cost and the accessibility measure in Georgia. Here we take a closer look at Atlanta and its surrounding regions. Throughout Atlanta, the travel cost has decreased in the past 13 years with a significant decrease in the northern part. Except for low population regions, the travel cost is uniformly low. On the other hand, when accounting for population density, the access to financial services is predominantly low in the southern Atlanta, i.e. the accessibility measure is high. In addition, the service access has decreased in the southern Atlanta and increased in the northern Atlanta over the past 13 years.

On the other hand, from Figure 4, the northern part of the Atlanta is largely populated by White population along with a lower percentage of Hispanic population whereas the southern part of Atlanta is largely populated by Black population. Therefore, in Atlanta, there is lower accessibility to financial services for Black population than for Hispanic and White population. In addition, income distribution is not uniform, with a patch of high income neighborhoods in the middle of Atlanta whereas both southern and northern Atlanta have large clusters of low-medium income population. Contrasting the income distribution to the accessibility to financial services, we can identify potential business opportunities for financial services in the southern neighborhoods of Atlanta. 


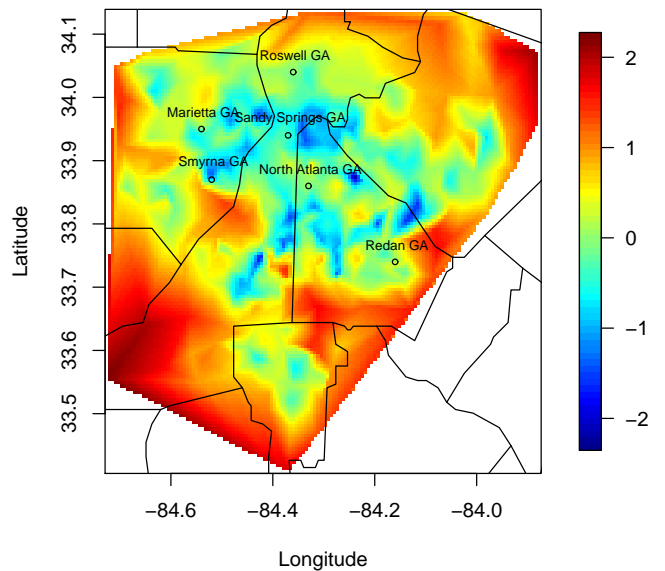

(a)

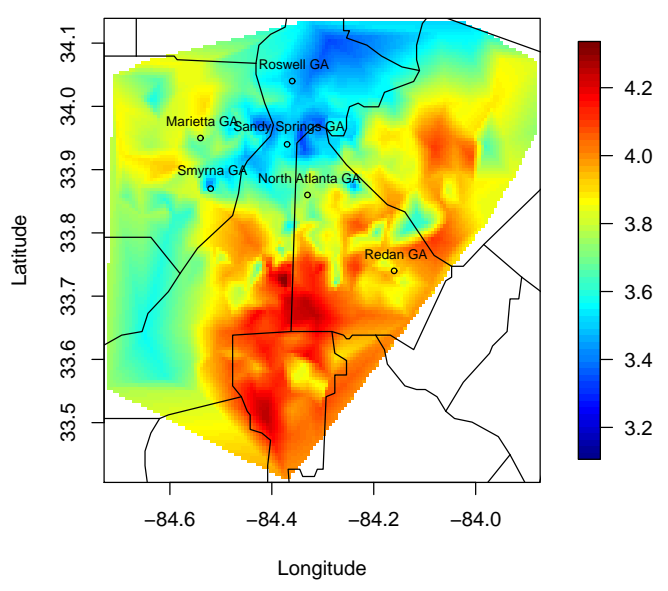

(c)

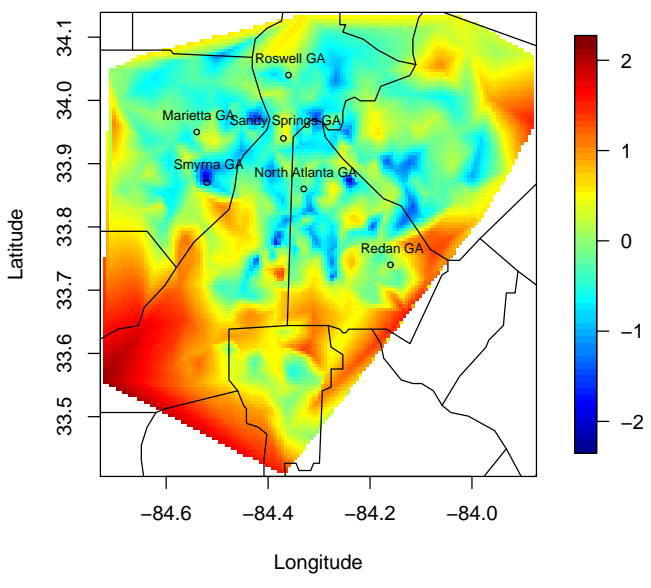

(b)

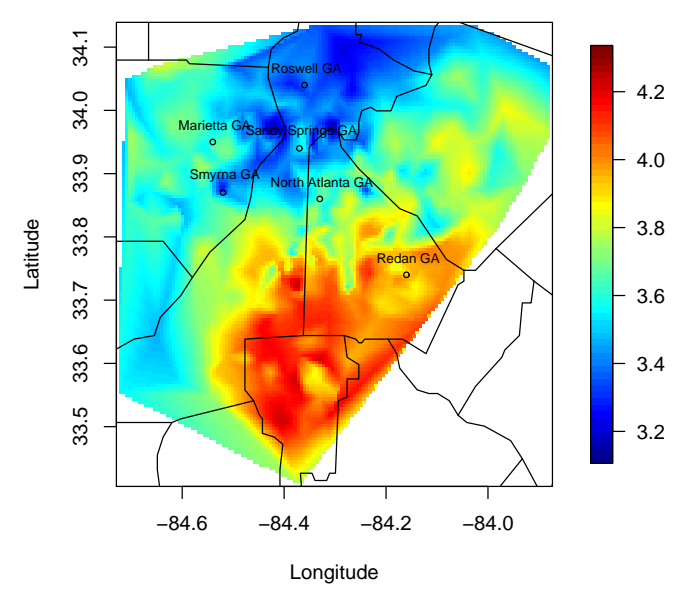

(d)

Figure 3: Atlanta: (a) The travel cost - 1996; (b) Travel Cost - 2008; (c) Accessibility -1996; and (d) Accessibility -2008. 


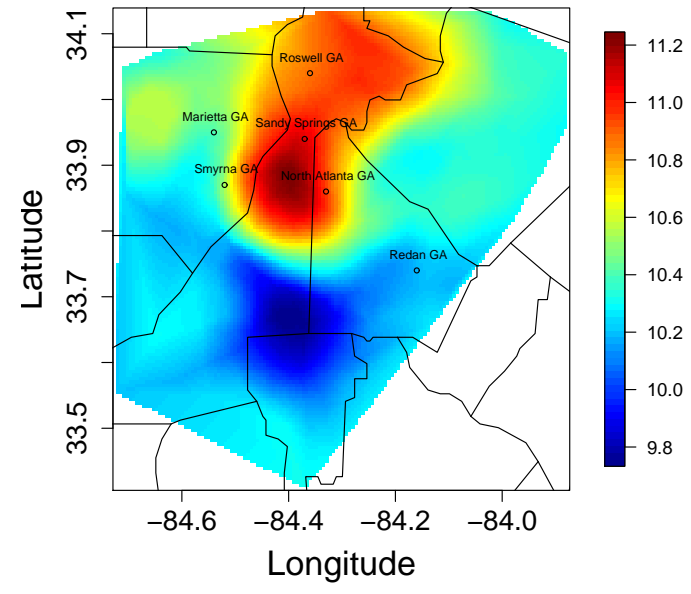

(a) Income

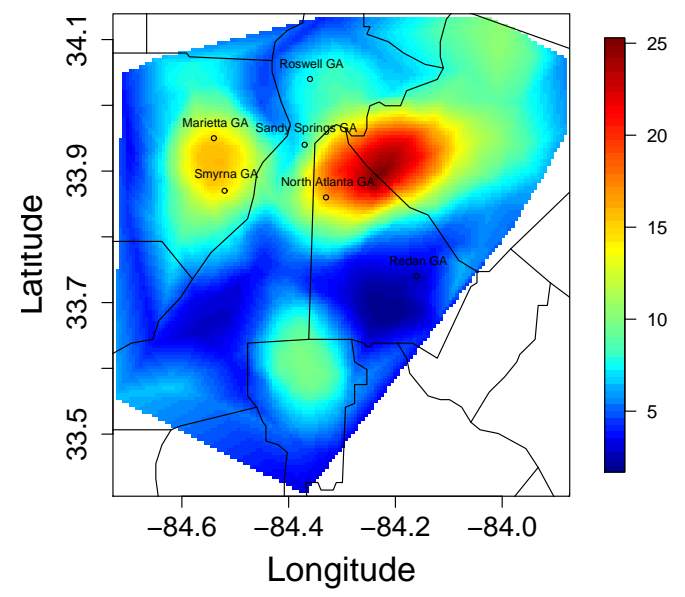

(c) $\%$ of Hispanic population

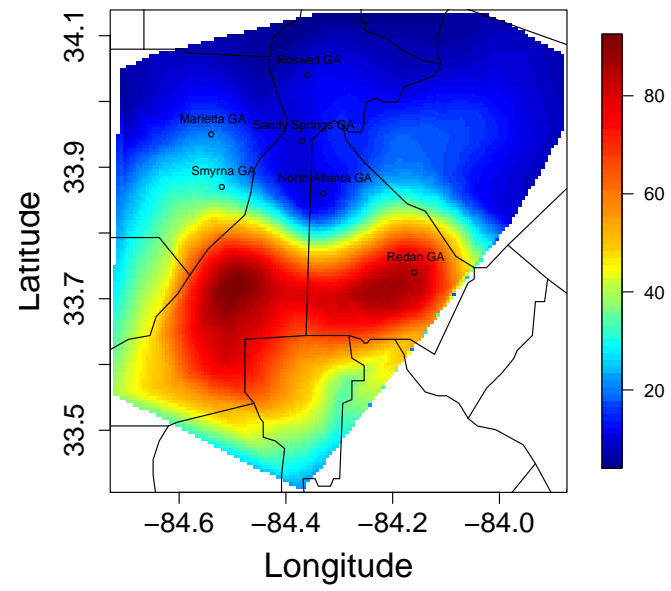

(b) $\%$ of Black population

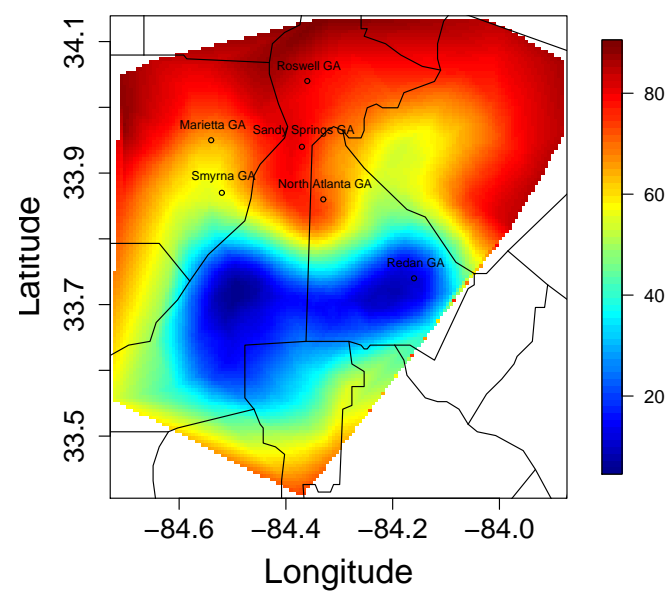

(d) $\%$ of White population

Figure 4: Demographic variables in Atlanta, GA in 2006. 


\section{Supplemental Material 7: Case Study}

Hierarchical Varying Coefficient Model: Results and Findings in Georgia. This supplemental material provides additional figures to complement the discussion on the results and findings from the application of the hierarchical varying coefficient model. Specifically, we include here the spatial and temporal regression coefficients corresponding to five large banks in our study: BOA, BB\&T, Regions, SunTrust and Wachovia (now Wells Fargo).

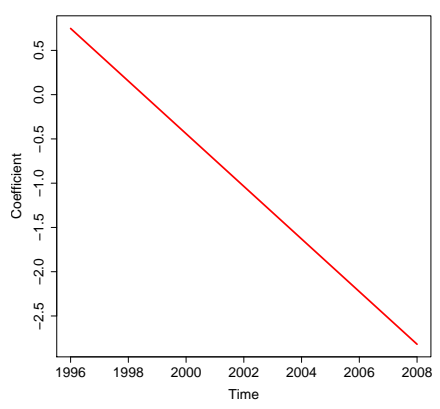

(a) BOA: $\eta_{\text {income }}(t)$

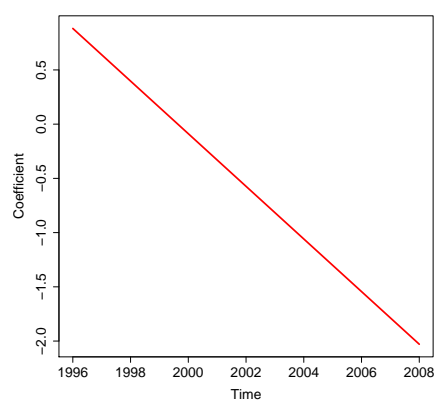

(b) B\&BT: $\eta_{\text {income }}(t)$

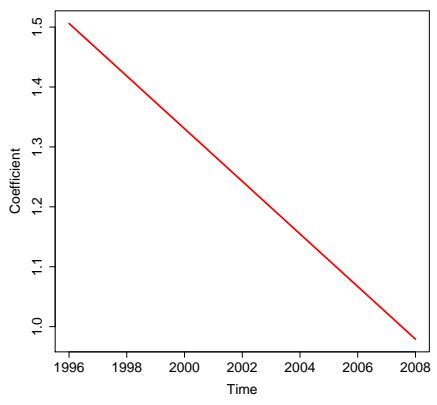

(c) Regions: $\eta_{\text {income }}(t)$

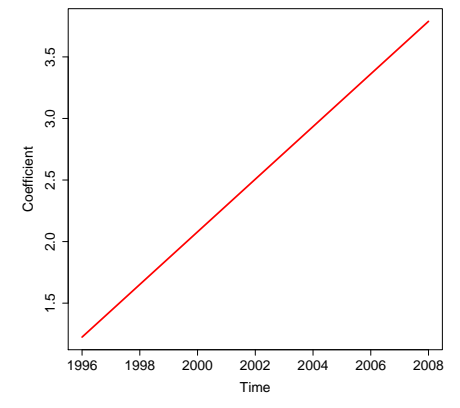

(d) SUNTrust: $\eta_{\text {income }}(t)$

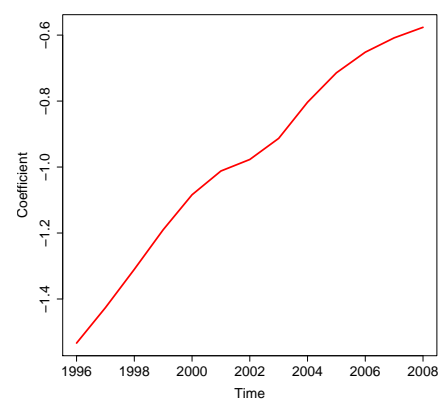

(e) Wachovia: $\eta_{\text {income }}(t)$

Figure 5: The time-varying deviation patterns for the income level. 


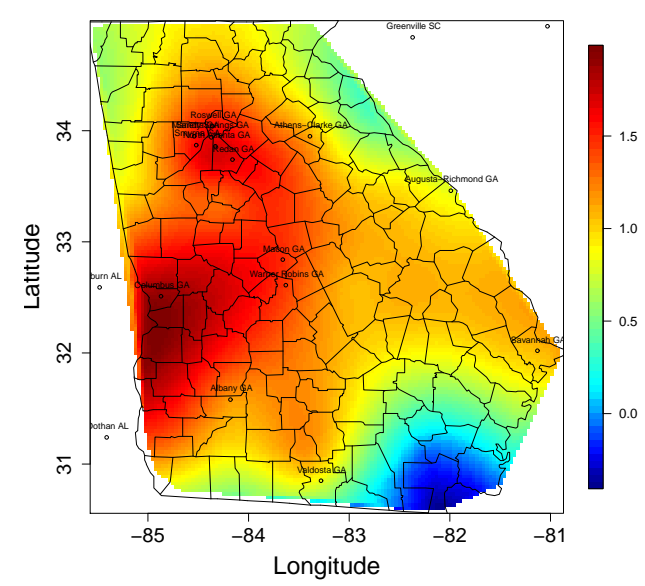

(a) Accessibility vs. Income in 2008

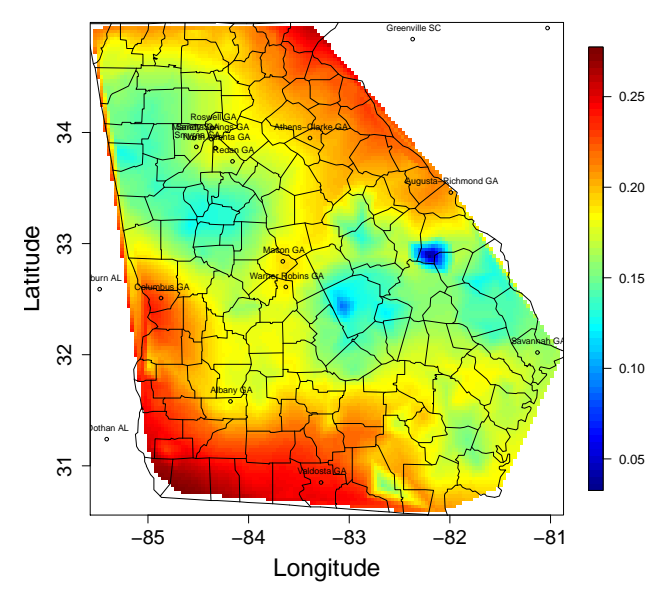

(c) Accessibility vs. Hispanic in 2008

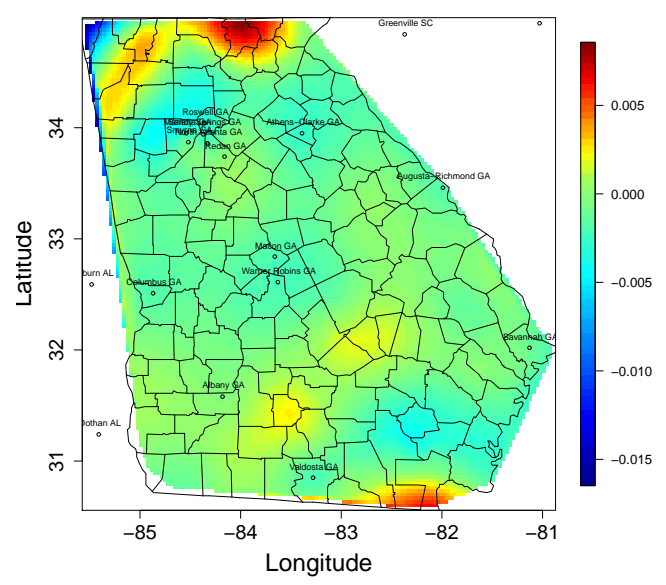

(b) Accessibility vs. Black in 2008

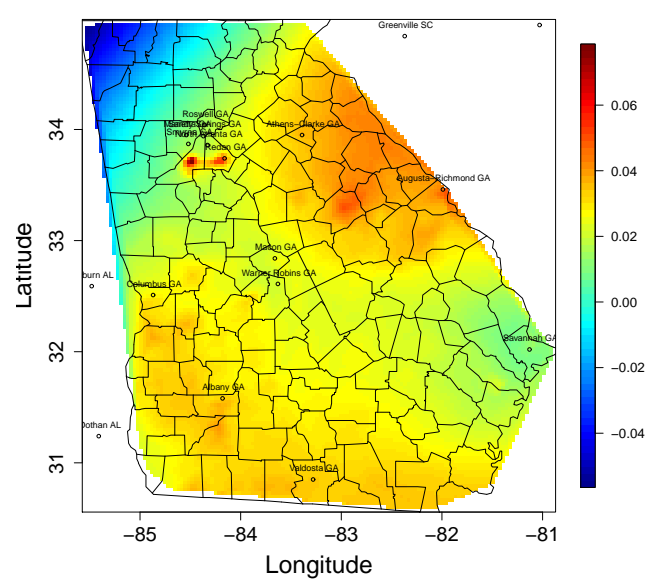

(d) Accessibility vs. White in 2008

Figure 6: Bank of America in Georgia: The time-varying coefficients (a)-(d) and the spatial relationship pattern in 2008 for four demographic covariates - income, percentage of Black, Hispanic and White population. 


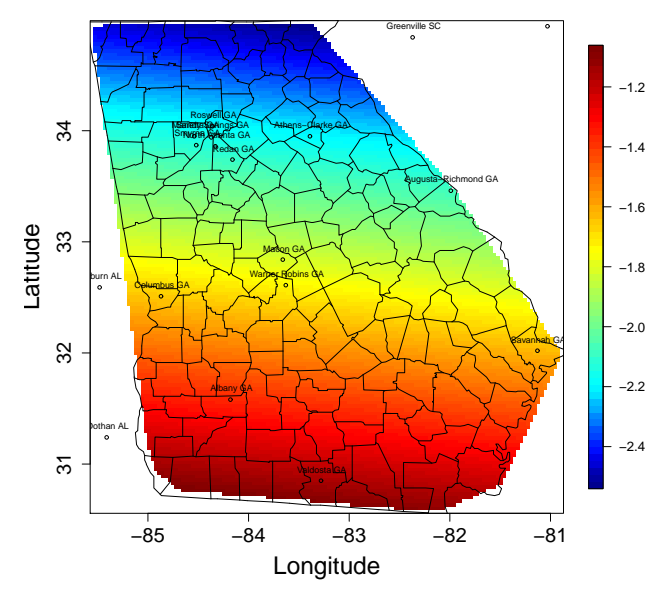

(a) Accessibility vs. Income in 2008

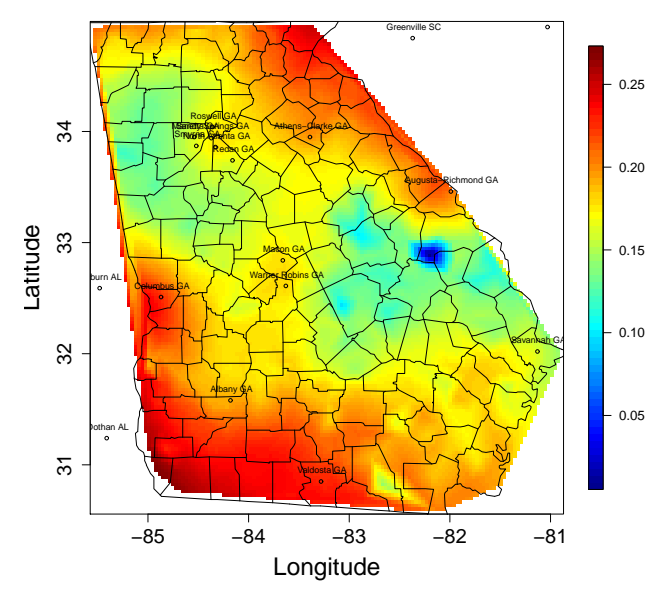

(c) Accessibility vs. Hispanic in 2008

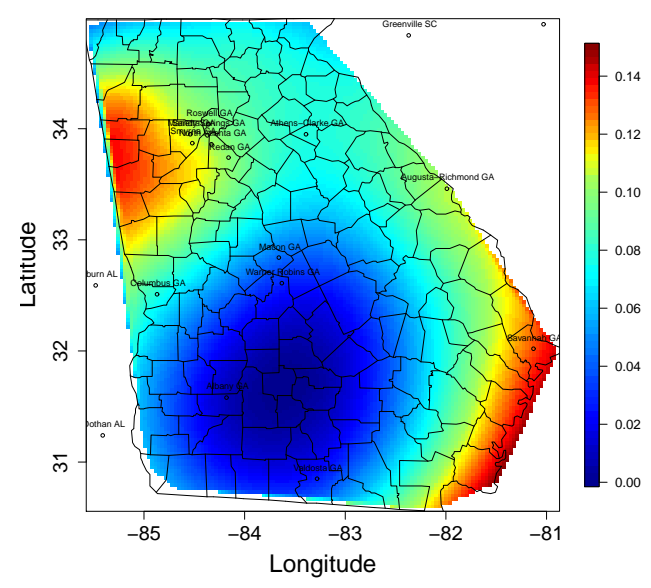

(b) Accessibility vs. Black in 2008

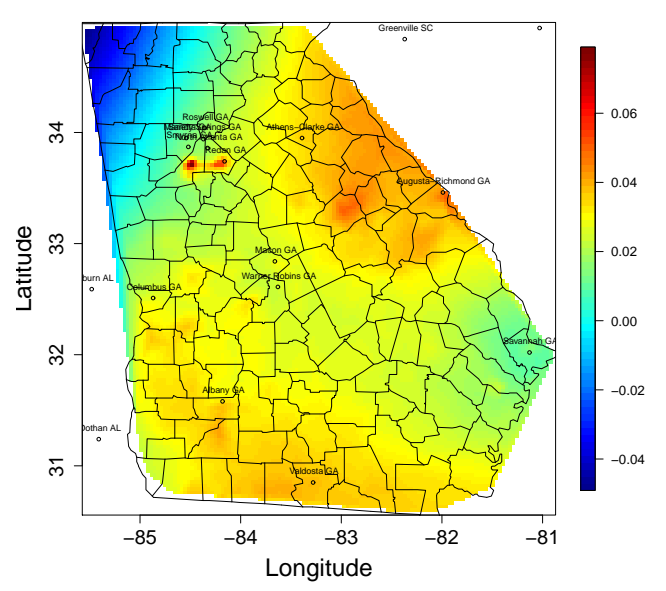

(d) Accessibility vs. White in 2008

Figure 7: Brunch Banking and Trust Company in Georgia: The time-varying coefficients (a)-(d) and the spatial relationship pattern in 2008 for four demographic covariates - income, percentage of Black, Hispanic and White population. 


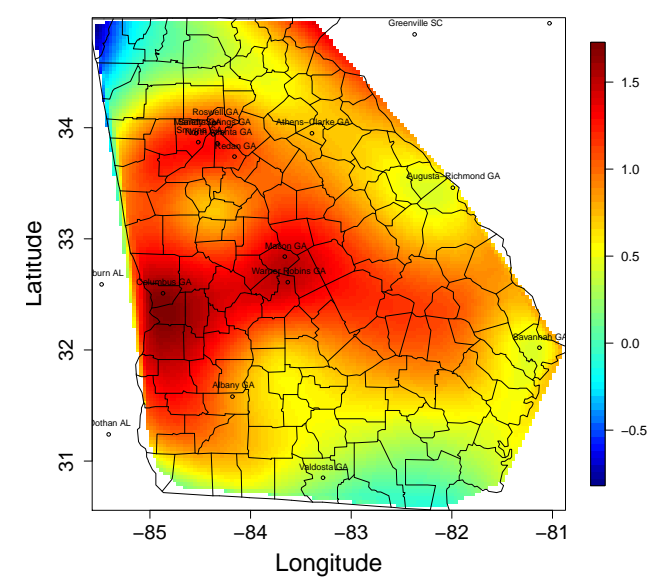

(a) Accessibility vs. Income in 2008

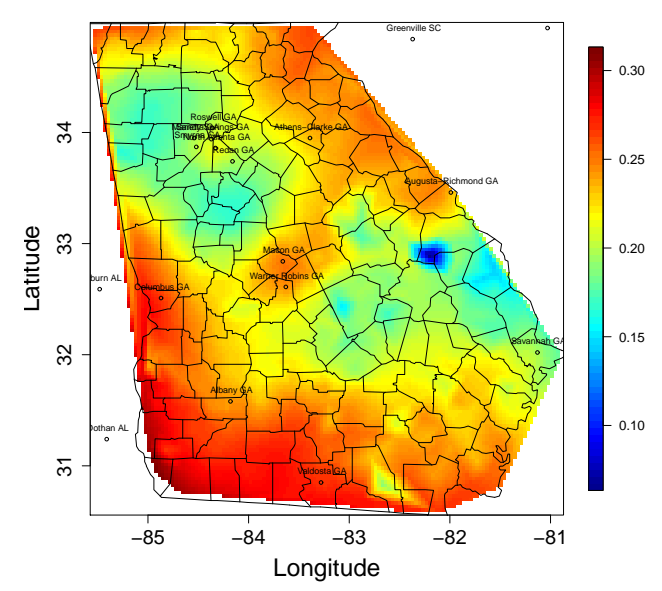

(c) Accessibility vs. Hispanic in 2008

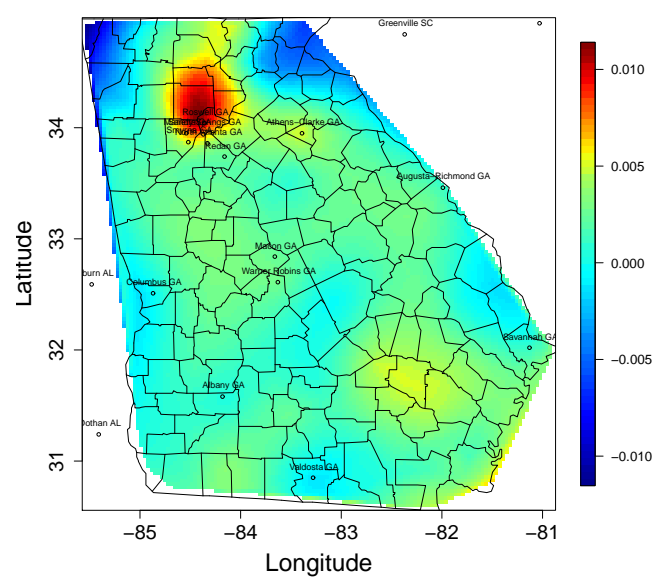

(b) Accessibility vs. Black in 2008

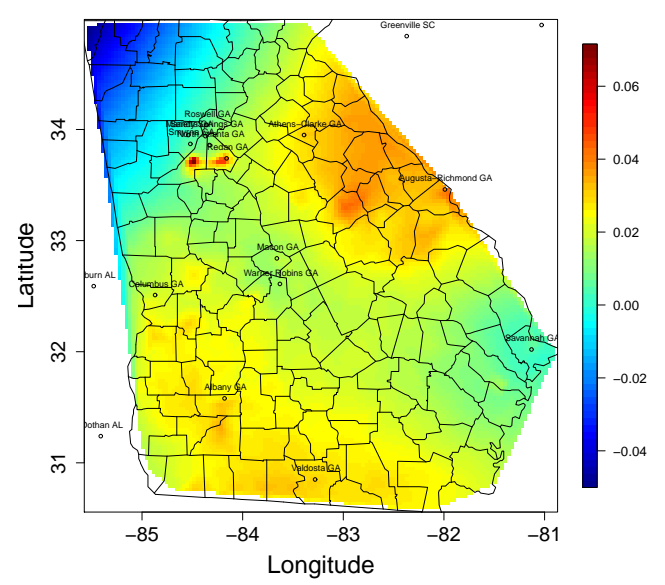

(d) Accessibility vs. White in 2008

Figure 8: Regions in Georgia: The time-varying coefficients (a)-(d) and the spatial relationship pattern in 2008 for four demographic covariates - income, percentage of Black, Hispanic and White population. 


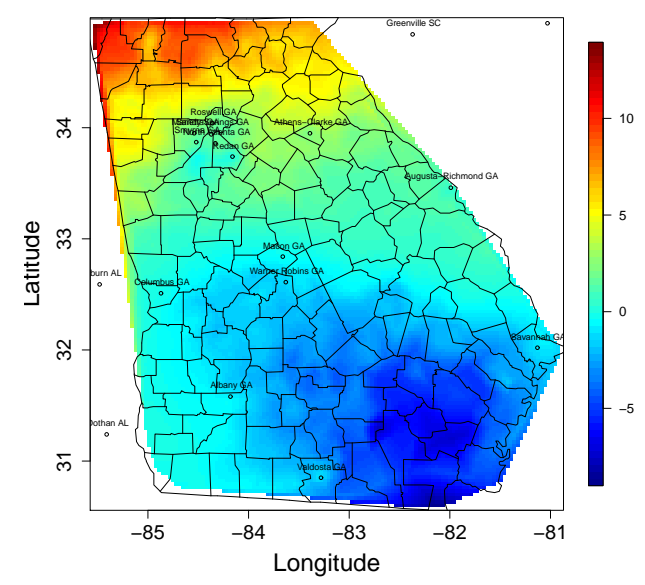

(a) Accessibility vs. Income in 2008

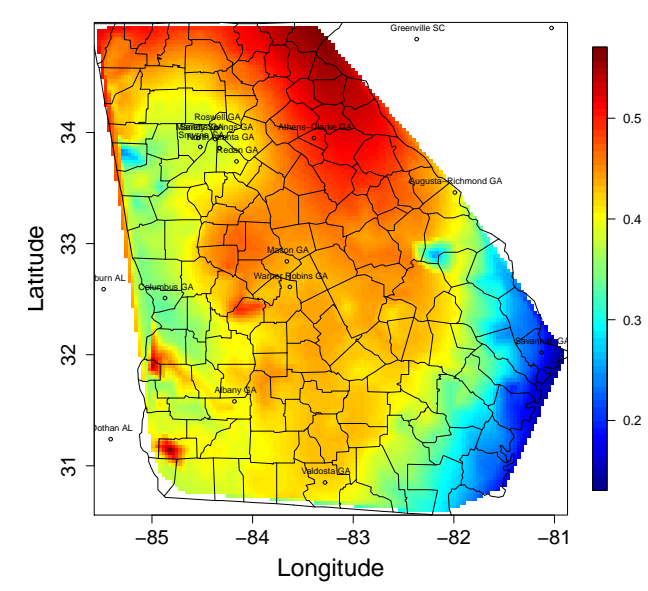

(c) Accessibility vs. Hispanic in 2008

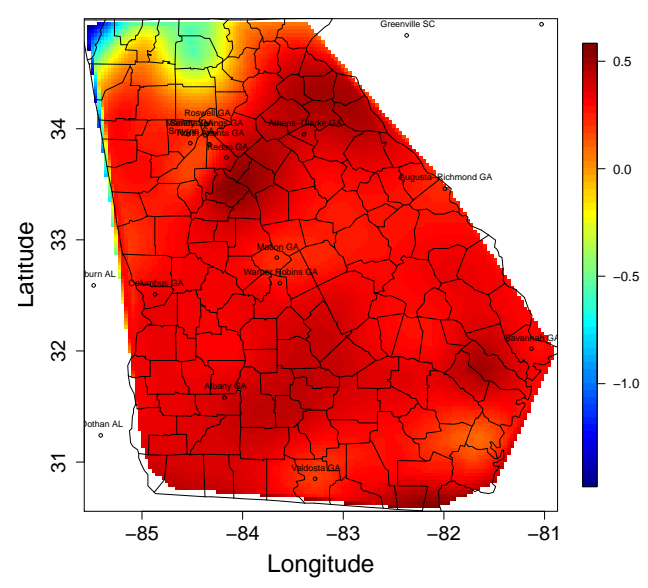

(b) Accessibility vs. Black in 2008

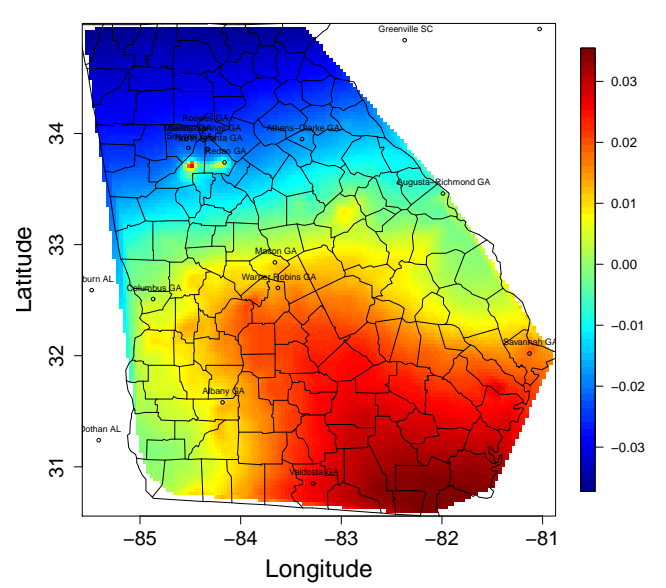

(d) Accessibility vs. White in 2008

Figure 9: SUN Trust in Georgia: The time-varying coefficients (a)-(d) and the spatial relationship pattern in 2008 for four demographic covariates - income, percentage of Black, Hispanic and White population. 


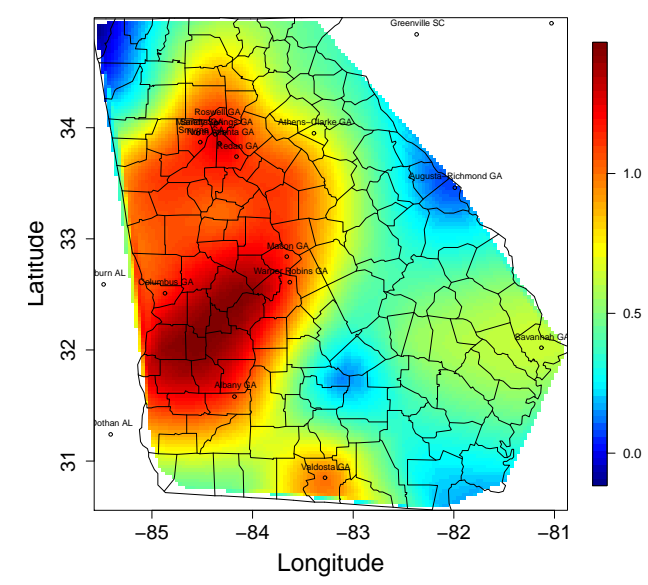

(a) Accessibility vs. Income in 2008

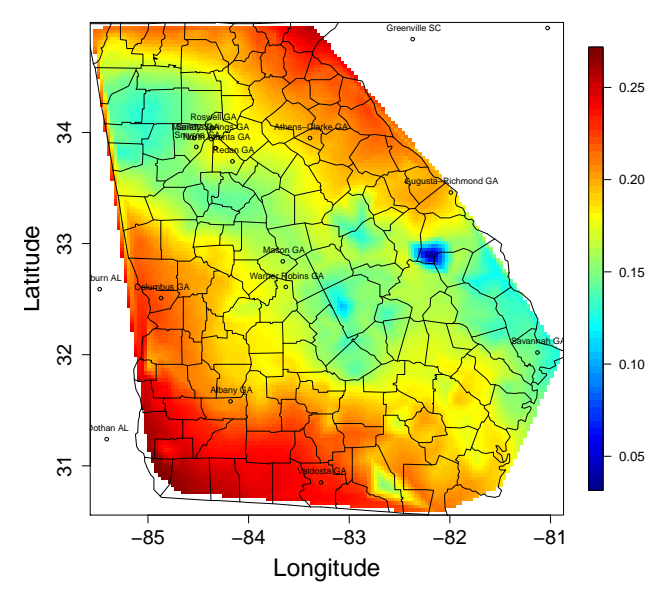

(c) Accessibility vs. Hispanic in 2008

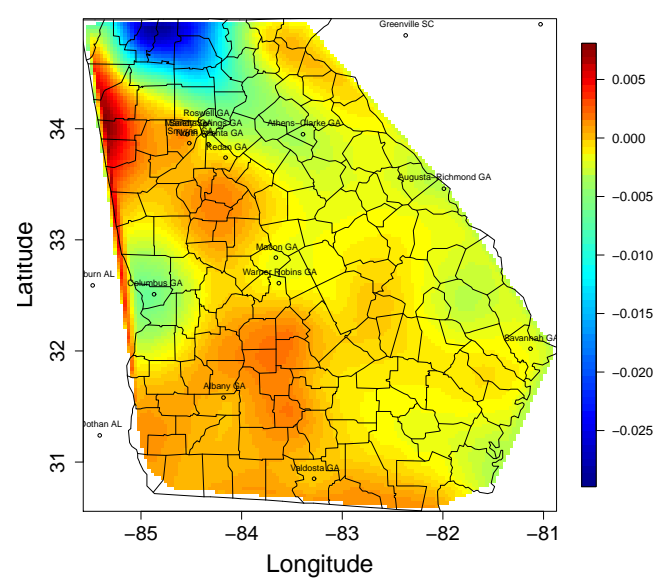

(b) Accessibility vs. Black in 2008

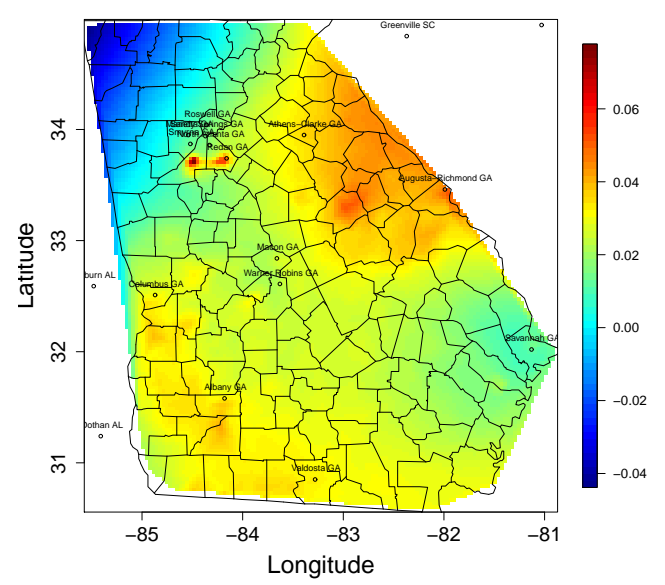

(d) Accessibility vs. White in 2008

Figure 10: Wachovia in Georgia: The time-varying coefficients (a)-(d) and the spatial relationship pattern in 2008 for four demographic covariates - income, percentage of Black, Hispanic and White population. 